\title{
Topography-Driven Shape, Spread, and Retention of Leaf Surface Water Impacts Microbial Dispersion and Activity in the Phyllosphere
}

\author{
Hung K. Doan, ${ }^{1}$ Viviane N. Ngassam, ${ }^{2}$ Sean F. Gilmore, ${ }^{3}$ Robin Tecon, ${ }^{1}$ Atul N. Parikh, ${ }^{2}$ and Johan H. J. Leveau ${ }^{1, \dagger}$ \\ ${ }^{1}$ Department of Plant Pathology, University of California, Davis, CA, U.S.A. \\ ${ }^{2}$ Department of Biomedical Engineering and Department of Materials Science and Engineering, University of California, Davis, CA, U.S.A. \\ ${ }^{3}$ Department of Applied Science, University of California, Davis, CA, U.S.A.
}

Accepted for publication 12 May 2020.

\section{ABSTRACT}

To study the impact of lateral variation in surface topography on the microbial colonization of plant leaves, we used a two-step casting process to generate topomimetic "replicasts" in polydimethylsiloxane which faithfully resembled leaf surface topography at submicrometer scale. This process revealed that the shape and size of the phyllotelma - the collective body of standing water on a leaf surface-was a function of wetting method and presence of surface features, in particular leaf veins. The use of dyes and fluorescently labeled bacteria showed that these veins, especially on the abaxial side, contributed to the pooling, retention, and lateral spread of water and microorganisms. Bodies of water along veins acted as conduits that allowed bacterial cells to sense chemicals over distances exceeding their body length by four orders of magnitude. Bacterial survival rates under conditions of low relative humidity were twice as high on leaf replicasts as on flat surfaces, suggesting topography-dependent protection from desiccation. Our findings highlight the need for greater quantitative understanding of the impact of leaf surface topography on phyllotelma connectivity and the outcomes of interactions between microorganisms and with their plant host. Such understanding benefits both theory and practice of phyllosphere microbiology in areas such as foliar disease management and food safety of leafy greens.

Keywords: bacteriology, biomimetic, ecology, habitat fragmentation, microorganism, epiphytic, PDMS, phylloplane, phyllotelma, rhizosphere, phyllosphere, soft lithography, surface roughness, surface wettability
The term phyllosphere (Ruinen 1956) refers to plant leaf surfaces as microbial biomes, or "theaters of microbial activity" (Whipps et al. 1988). To its microscopic inhabitants, a leaf surface represents an environment that is highly heterogeneous in terms of physical, chemical, and biological attributes such as landscape topography (Barthlott et al. 2017; Mechaber et al. 1996), nutrient availability (Farhan Ul Haque et al. 2017; Leveau and Lindow 2001a; RemusEmsermann et al. 2011; Ryffel et al. 2016), and microbial

\section{Corresponding author: J. H. J. Leveau; jleveau@ucdavis.edu}

Funding: The research reported here was made possible by funding from USDANIFA-AFRI through grant number 2013-02075 to J. H. J. Leveau.

Current address of S. F. Gilmore: Lawrence Livermore National Laboratory, Livermore, CA 94550, U.S.A.

Current address of R. Tecon: Department of Environmental Systems Science, ETH Zürich, Zürich, Switzerland.

*The $\boldsymbol{e}$-Xtra logo stands for "electronic extra" and indicates there are supplementary materials and supplementary figures published online.

The author(s) declare no conflict of interest.

(C) 2020 The American Phytopathological Society distributions and population densities (Monier and Lindow 2004, 2005). Although it may be hard to measure heterogeneity at this scale (Leveau et al. 2018), it is easy to imagine how microheterogeneity at the leaf surface might affect the fate of individual microbial immigrants and colonizers, even on the same leaf (Leveau 2019; Remus-Emsermann and Leveau 2010; Remus-Emsermann and Schlechter 2018). An improved understanding of the causes and consequences of small-scale heterogeneity in the phyllosphere may help us better interpret and predict large-scale phenomena such as those that relate to plant disease caused by foliar pathogens (Garin et al. 2018).

Recently (Doan and Leveau 2015), the term "phyllotelma" was introduced to refer to the collective body of water that is present on a leaf surface. The phyllotelma signifies a compartment of the phyllosphere that is different from but intimately connected to the phylloplane (i.e., the leaf surface). Size and shape of the phyllotelma are the combined result of multiple processes (Beattie 2011; Vacher et al. 2016), including precipitation (e.g., rain, fog, and irrigation), condensation (e.g., dew from air or soil), recondensation of transpired water vapor (Burkhardt et al. 1999), run-off, evaporation (Zhu et al. 2008), and guttation (Singh 2016). Free water on the leaf surface is essential for the survival, growth, and activity of phyllospheric microorganisms (Beattie 2011). Surface water allows 
microorganisms to relocate laterally (Tecon and Leveau 2012), acquire nutrients leached from the leaf interior (van der Wal and Leveau 2011), and communicate with other microorganisms (Dulla and Lindow 2008). Surface water offers foliar pathogens better prospects of multiplying and causing disease (Rowlandson et al. 2015). As water evaporates from the leaf surface, microbial populations typically decline in size (Wilson and Lindow 1992) and trigger behaviors to avoid desiccation such as the production of osmoprotectants (Bonaterra et al. 2007; Scott et al. 2017) and biofilms (Bogino et al. 2013). The microbial experience of water availability in the phyllosphere can vary substantially among cells of the same population, depending on the location of individual cells in relation to leaf surface structures. For example, bacterial cells close to the edge of a drying drop or further away from leaf veins experienced elevated levels of water stress (Axtell and Beattie 2002). These findings support the notion that leaf surface topography plays a role in shaping the phyllotelma and can thereby influence microbial survival and reproduction.

The size and shape of the phyllotelma are relatively well understood at the canopy and leaf level, where water can be seen with the naked eye and measured in quantitative terms such as leaf wetness (Zhu et al. 2008) and contact angle (Rowlandson et al. 2015). However, at scales that matter to microbial leaf colonizers, direct visualization and quantification of the phyllotelma, or "microscopic leaf wetness" (Burkhardt and Hunsche 2013), can be challenging. In light- or laser-based microscopy, cover slips and mounting media can interfere with the natural waterscape on the leaf surfaces. Also, the heat of lights or lasers can alter the phyllotelma and damage the leaf surface. Electron microscopy involves sample preparations and ultrahigh vacuum conditions that are generally incompatible with maintaining the natural state of the phyllotelma. However, techniques such as cryoscanning electron microscopy and environmental scanning electron microscopy (Gorb et al. 2007; Zheng et al. 2008) have been successful in capturing the phyllotelma at micrometer dimensions. The complex collection of variably sized drops and other shapes of water that these studies typically reveal pose a number of general questions relevant to phyllosphere microbiology. (i) How many and what kind of microorganisms might each one of these drops support? (ii) What are the conditions within these individual bodies of water and how different are they between drops? (iii) To what extent are water drops connected to each other by threads of water that follow the contours of the leaf surface and are difficult to visualize? We argue that an understanding of the connectivity and fragmentation of the phyllotelma, as a function of leaf topography, can inform how the water landscape on the leaf surface facilitates or constrains the interactions of microbes with each other and with their host, and how this scales up to macroscale observations, reflecting the emergent collective behaviors of microorganisms at the leaf surface.

Here, we explore the use of artificial leaf surfaces for the quantitative study of the phyllotelma in relation to the local variation in leaf surface topography and its impact on phyllospheric microbiology. More specifically, we describe a casting process that uses the silicon-based, elastomeric polymer, polydimethylsiloxane (PDMS), in a two-step casting protocol to replicate leaf surface topography down to nanometer resolution, as others have done previously (Schulte et al. 2009; Soffe et al. 2019b; Sun et al. 2005, 2019; Wu et al. 2016; Zhang et al. 2014). Such replicated leaf surfaces (or "leaf replicasts") have been referred to as "biomimetic" (Zhang et al. 2014) but, given that only the surface structure is replicated, "topomimetic" is a more accurate term. As topomimetic surfaces, these PDMS leaf replicasts represent synthetic environments that allow a reductionist approach to studying the complexity of the phyllosphere, with emphasis on the role of surface topography. Like fresh leaves, PDMS replicasts can be inoculated, incubated, and assessed by microscopy or surface washing for microbial colonization patterns (Soffe et al. 2019b; Zhang et al. 2014). They also have been used to show how water retention of colloidal particles correlates with leaf roughness (Sun et al. 2019). As a material of choice, PDMS offers many benefits: it accurately and stably replicates leaf microstructures, its structural durability allows examination by various kinds of microscopy, its surface wettabilities are comparable with those of fresh leaves, and it does not seem to interfere with microbial viability (Soffe et al. 2019a,b; Zhang et al. 2014). Here, we exploited these properties to quantify the phyllotelma as a function of leaf surface topography and as a factor contributing to bacterial survival, growth, retention, and perception of the leaf environment; and to show an additional benefit of PDMS, which is the ability to replicate accurately the shape and size of waterscapes on wet leaves.

\section{MATERIALS AND METHODS}

Leaf surface replicasting. As a source of leaves, we used plants grown in the greenhouse in Sunshine mix number 1 (Sun Gro Horticulture, Bellevue, WA, U.S.A.) with $10 \mathrm{~h}$ of supplemental light (provided by high-pressure sodium light bulbs), at 27 to $30^{\circ} \mathrm{C}$ during the day and 18 to $21^{\circ} \mathrm{C}$ at night: Phaseolus vulgaris L. (green snap bean, variety Blue Lake 274), Lactuca sativa L. (lettuce, variety Parris Island Cos), Solanum lycopersicum L. (tomato, variety Big Boy), S. tuberosum L. (potato, variety Yukon Gold), Spinacia oleracea L. (spinach, variety Tyee), and Calathea zebrina (zebra plant, variety Humilior). When referring to lettuce and spinach leaves at different developmental growth stages, we use here the relative Biologische Bundesanstalt, Bundessortenamt and Chemical Industry (BBCH) scales (Meier 2001) in addition to the absolute age of the plant. From the plants listed above, leaves were used in a two-step replica casting process (Supplementary Fig. S1) to produce topomimetic leaves (i.e., reproductions of plant leaf topography in PDMS). We also refer to these as leaf replicasts. This process, which generates a positive PDMS replicast from a negative PDMS mold that itself is a replicast from a fresh leaf surface, is described in detail in the Supplementary Information. Up to 10 positive replicasts were prepared from a single negative mold. Flat PDMS surfaces (i.e., lacking any topography) were generated using Petri dishes instead of fresh plant leaves as a mold (see Supplementary Information for details). PDMS replicasts were stored in Parafilmed Petri dishes until use.

PDMS replicasts of wetted leaves. In a variation on the standard leaf replicasting protocol, we used surface-wetted leaves as starting material to capture, visualize, and quantify the water landscape (phyllotelma) on leaf surfaces. For this purpose, primary leaves of 30-day-old green snap bean plants were wetted with Milli-Q water (Millipore Corporation, Billerica, MA, U.S.A.) in three different ways: (i) brief immersion (dip); (ii) spray from a distance of approximately $25 \mathrm{~cm}$ with squirts from a common spray bottle (Impact, American Fork, UT, U.S.A.) (coarse spray; average droplet diameter of $85.4 \pm 15.0 \mu \mathrm{m}$ after spraying on a flat PDMS surface); and (iii) spray from a distance of approximately $25 \mathrm{~cm}$ using an airbrush (Preval, Coal City, IL, U.S.A.) (fine spray; average diameter of $30.3 \pm 7.3 \mu \mathrm{m}$ after spraying on a flat PDMS surface). Following application of the water, leaves (still wet) were used to generate a negative PDMS mold and then a positive PDMS replicast, using the protocol described under Supplementary Information.

Microscopy. Sections of fresh leaves or PDMS replicasts of dry or wetted leaves were visualized directly at various magnifications using an Axio Imager M2 (Zeiss, Jena, Germany) equipped with an 
AxioCam MRm (Zeiss) camera, or a 3D Laser Scanning Confocal Microscope VK-X (Keyence, Osaka, Japan). For scanning electron microscopy (SEM), fresh leaf samples were fixed overnight in Karnovsky's fixative $(2.5 \%$ [vol/vol] paraformaldehyde and $2.0 \%$ [vol/vol] glutaraldehyde in $0.08 \mathrm{M}$ sodium phosphate buffer, $\mathrm{pH}$ 7.2), rinsed three times for $5 \mathrm{~min}$ in $0.1 \mathrm{M}$ sodium phosphate buffer, and dehydrated in ascending concentrations of ethanol (30, 50, 70, 95 , and $100 \%$ [vol/vol]) for $15 \mathrm{~min}$ at each concentration, then dried in a Samdri-PVT-3D critical point dryer (Tousimis, Rockville, MD, U.S.A.). Dehydrated leaf samples, or sections of PDMS replicasts, were mounted on aluminum stubs (Ted Pella Inc., Redding, CA, U.S.A.) using double-sided carbon tape (Ted Pella Inc.) and goldcoated for $60 \mathrm{~s}$ in a Pelco Auto Sputter Coater SC-7 (Ted Pella Inc.). SEM images were taken using a Phenom Pro (Phenom-World, Eindhoven, The Netherlands) with a $5-\mathrm{kV}$ accelerating voltage. As needed, microscope images were captured and analyzed using Zeiss or Keyence software or ImageJ (https://imagej.nih.gov/ij/)) to quantify the surface area or perimeter of leaf structures, including veins, pavement cells, hooked trichomes, glandular trichomes, and stomata, as well as individual bodies of water. Circularity (C) of bodies of water was calculated as the square root of $4 \pi \mathrm{A} / \mathrm{P}^{2}$, where $\mathrm{A}$ is the area and $\mathrm{P}$ the perimeter of each body of water (Olson 2011).

Characterization of PDMS leaf replicasts. To test the tolerance of PDMS replicasts to various microbial decontamination procedures (required for reuse of PDMS replicasts), 10-mm-diameter discs were excised from PDMS replicasts with a cork borer and either autoclaved for $30 \mathrm{~min}$ at $121^{\circ} \mathrm{C}$ or submerged for $12 \mathrm{~h}$ at $23^{\circ} \mathrm{C}$ in $0.1 \%$ ( $\mathrm{vol} / \mathrm{vol})$ sodium hypochlorite $(\mathrm{NaOCl}), 10 \%$ ( $\mathrm{vol} / \mathrm{vol})$ lactic acid, or $10 \%(\mathrm{vol} / \mathrm{vol})$ phosphoric acid $\left(\mathrm{H}_{3} \mathrm{PO}_{4}\right)$. Microscopic images of the surface were then taken and compared with those of nontreated surfaces.

To compare the hydrophobicity of fresh leaves and PDMS replicasts, we deposited onto each one of three fresh bean leaves, three PDMS bean leaf replicasts, and three flat PDMS replicasts (controls) six 15- $\mu$ l drops of Milli-Q water with or without $0.2 \%$ (vol/vol) Silwet L77 (Helena Chemical Company, Collierville, TN, U.S.A.) at arbitrary adaxial or abaxial locations. For each drop, the contact angle was measured using a Face CA-DT contact-angle analyzer (Kyowa Interface Co., Niiza, Japan). Averages and standard deviations were calculated from the means of six drops per leaf, with three leaves representing each treatment.

The topography-dependent surface spread of water on fresh leaves and PDMS replicasts was measured by depositing $1-\mu \mathrm{l}$ drops of water (approximately 25 drops per replicast) containing $0.0005 \%$ (vol/vol) brilliant cresyl blue (Sigma-Aldrich) and $0.2 \%$ (vol/vol) Silwet L77 at arbitrary locations on the PDMS replicasts of adaxial or abaxial bean leaf surfaces. Surfaces were photographed under the Axio Imager M2 microscope and, for each drop, the perimeter of its blue surface footprint was measured (in centimeters) and plotted as a function of its relative $x, y$ location on the leaf surface. Drops with overlapping footprints were excluded from the analysis. For each one of six leaf surfaces (three adaxial and three abaxial), the spread patterns (i.e., droplet perimeters) from 12 PDMS replicasts (identical to each other in topography but different in terms of the arbitrary placement of water droplets) were compiled and used to generate a heatmap that depicts local variation in the surface spread of water using Quantum GIS version, 2.18.3 (https://qgis.org). Flat PDMS surfaces were used as a control. For statistical analysis of the data, droplet perimeters from all 12 replicasts that represented one of the six leaf surfaces were averaged, and these six averages were compared to identify significant differences between adaxial and abaxial surfaces. To relate water spread to leaf topography, we averaged and compared for each one of the six leaf surfaces (three abaxial and three adaxial) the perimeters of two subsets of droplets: one subset of droplets inside two opposing quadrants of a diamondshaped projection onto the central part of the PDMS replicast (these two quadrants both encompassed portions of the primary vein) and another subset of droplets inside the other two quadrants of that diamond (these quadrants lacked the primary vein).

Bacterial strains. All bacterial strains used in this study were stored at $-80^{\circ} \mathrm{C}$ in $10 \%$ glycerol and routinely grown at $28^{\circ} \mathrm{C}$ in lysogeny broth (LB), in M9 minimal medium supplemented with an appropriate carbon source, or on tryptic soy agar (TSA) or LB agar $(1.5 \%)$ plates, amended with antibiotics, as appropriate, to final concentrations as follows: kanamycin at $50 \mathrm{mg} /$ liter, rifampicin at $50 \mathrm{mg} /$ liter, or tetracycline at $10 \mathrm{mg} /$ liter. Pantoea agglomerans 299R (Pa299R) is a rifampicin-resistant derivative of the phyllosphere model bacterium Erwinia herbicola 299 (Brandl et al. 1996). Strain Pa299R::JBA28 (Leveau and Lindow 2001b) is a kanamycin-resistant variant of Pa299R carrying a chromosomal insertion of an S2R-modified version of the gfpmut3 gene and expressing stable green fluorescent protein (GFP) from LacIrepressible promoter $\mathrm{P}_{A 1 / O 4 / O 3}$. Strain Pa299R::JBA28 lacks a gene for $L a c$; thus, this strain constitutively expresses GFP. Cells of Pa299R::JBA28 carrying plasmid pCPP39 (Leveau and Lindow $2001 b$ ) produce GFP in response to the presence of isopropyl- $\beta-D-$ thiogalactopyranosid (IPTG; Sigma-Aldrich), because pCPP39 harbors an lacI gene. Plasmid pCPP39 also confers resistance to tetracycline. Escherichia coli ATCC 700728 (Ec70078) is a nontoxigenic (confirmed lack of Shiga-toxin production genes) BSL-1 rifampicin-resistant derivative of a natural $\mathrm{O} 157: \mathrm{H7}$ isolate (Moyne et al. 2011); it was provided by Dr. Linda Harris, Department of Food Science and Technology, University of CaliforniaDavis. Bacillus amyloliquefaciens subsp. plantarum strain FZB42 (BaFZB42) was obtained from the Bacillus Genetic Stock Center (http://www.bgsc.org).

Bacterial growth conditions. To prepare Pa299R or Ec70078 bacteria for a typical leaf inoculation experiment, a single bacterial colony was transferred from agar medium into $10 \mathrm{ml}$ of M9 minimal medium supplemented with $0.4 \%$ glucose or LB plus appropriate antibiotics and incubated on a rotary shaker $(250 \mathrm{rpm})$ for $10 \mathrm{~h}$ at $28^{\circ} \mathrm{C}(\mathrm{Pa} 299 \mathrm{R})$ or $37^{\circ} \mathrm{C}(\mathrm{Ec} 70078)$ in the dark. In all, $200 \mu \mathrm{l}$ of this culture were transferred into $20 \mathrm{ml}$ of fresh M9+glucose medium or $\mathrm{LB}$ and incubated on a rotary shaker $(250 \mathrm{rpm})$ for $6 \mathrm{~h}$ at $28^{\circ} \mathrm{C}$ or $37^{\circ} \mathrm{C}$ in the dark to obtain an optical density at $600 \mathrm{~nm}\left(\mathrm{OD}_{600}\right)$ of 1 to 1.5 . Bacterial cells were harvested by centrifugation at $2,500 \times g$ for $10 \mathrm{~min}$, rinsed twice with Milli-Q water, and resuspended in M9+glucose medium or antibiotics as appropriate.

To generate a $B a \mathrm{FZB} 42$ spore suspension, a single colony of BaFZB42 was transferred into $100 \mathrm{ml}$ of Schaeffer's sporulation medium (Schaeffer et al. 1965) and incubated on a rotatory shaker at $37^{\circ} \mathrm{C}$ and $200 \mathrm{rpm}$ for $96 \mathrm{~h}$. Endospores were collected by centrifugation at 8,000 rpm for $20 \mathrm{~min}$ and resuspended in $100 \mathrm{ml}$ of Tris $\mathrm{HCl}(50 \mathrm{mM}, \mathrm{pH} 8.0)$ amended with $100 \mu \mathrm{l}$ of lysozyme (Sigma-Aldrich) at $10 \mathrm{mg} / \mathrm{ml}$ and incubated on a rotary shaker at $200 \mathrm{rpm}$ for $2 \mathrm{~h}$ at $37^{\circ} \mathrm{C}$ to lyse remaining vegetative cells. After centrifugation at $2,500 \times g$ for $10 \mathrm{~min}$, the supernatant was discarded and the spore pellet was rinsed twice and resuspended in Milli-Q water or M9 medium.

Water- and topography-dependent dispersal of chemicals along the leaf surface. To quantify the water- and topographydependent landscape connectivity on PDMS leaf replicasts, we placed $1-\mu 1$ droplets of a $100 \mathrm{mM}$ solution of IPTG either right next to $(0 \mathrm{~mm})$ or away from $(2,4$, or $8 \mathrm{~mm})$ a secondary vein on PDMS replicasts of bean abaxial leaves and let them dry for $2 \mathrm{~h}$ at $23^{\circ} \mathrm{C}$. These PDMS replicasts were produced from the abaxial sides of 18 different bean leaves, with 4 identical replicasts per leaf, for a total 
of 72 replicasts. Onto each replicast, four IPTG drops were spotted, each one at a different secondary vein, and each one at a different distance from the vein (i.e., $0,2,4$, or $8 \mathrm{~mm}$ ), for a total of $4 \times 72=$ 288 veins. Milli-Q water $(1 \mu \mathrm{l})$ amended with $0.2 \%$ Silwet $\mathrm{L} 77$ was then pipetted on top of each dried spot of IPTG and allowed to spread and dry for $2 \mathrm{~h}$ at $23^{\circ} \mathrm{C}$. Next, each vein $(n=288)$ was inoculated with a pattern of six $1-\mu l$ droplets containing a suspension $\left(10^{6}\right.$ bacteria/ml $)$ of IPTG-responsive Pa299R::JBA28 (pCPP39) in M9+glucose medium, each droplet between 0 and
$32 \mathrm{~mm}$ along the vein and between 0 and $8 \mathrm{~mm}$ away from the vein for a total of $288 \times 6=1,728$ bacterial drops (see Supplementary Figure S2 for details). Inoculated PDMS replicasts were placed on top of water-saturated germination paper (Anchor Paper Co.) in 100-mm-diameter plastic Petri dishes which were then Parafilmed and incubated for $12 \mathrm{~h}$ at $23^{\circ} \mathrm{C}$. Each bacterial spot was scored positive or negative for GFP expression by fluorescence microscopy. This entire experiment was repeated two times, for a total of three replicated experiments and $3 \times 1,728=5,184$ bacterial scores.
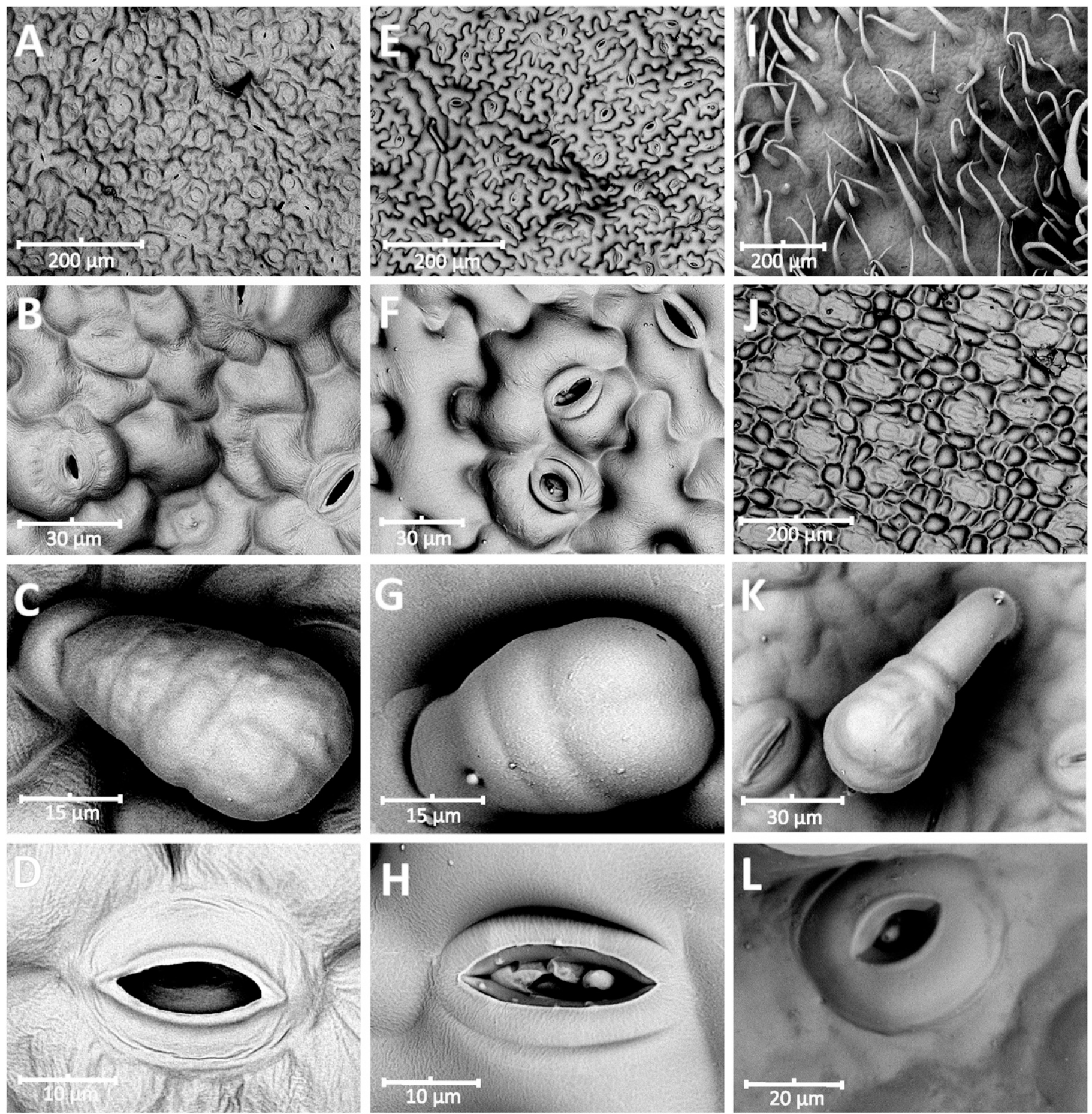

Fig. 1. Scanning electron microscopy images of $\mathbf{A}$ to $\mathbf{D}$, fresh bean leaves and $\mathbf{E}$ to $\mathbf{H}$, polydimethylsiloxane (PDMS) replicasts; as well as PDMS replicasts of I, potato; J, zebra plant, K, tomato; and L, lettuce leaves. All images represent abaxial leaf surfaces. Note the differences in scale between panels. 
For each combination of one of the four distances of IPTG from the vein (i.e., $0,2,4$, and $8 \mathrm{~mm}$ ) and 1 of the 12 possible parallel-to-thevein distances between IPTG and bacteria (i.e., 0, 2, 4, 6, 8, 12, 14, $16,24,28,30$, and $32 \mathrm{~mm}$ ), we plotted the incidence (percentage) of spots that scored positive for GFP for all three experiments combined. We also assessed whether differences in these incidences were statistically significant between the three repeated experiments (i) between distances of $0,2,4$, and $8 \mathrm{~mm}$ away from the vein versus along the vein and (ii) between distances parallel to the vein, either right next to the vein $(0 \mathrm{~mm})$ or away from the vein $(8 \mathrm{~mm})$. Flat PDMS surfaces were used as a control (these have no veins; thus, drops were placed in arbitrary locations).

Topography-dependent retention of water and bacteria on PDMS replicasts. To quantify water retention of PDMS replicasts as a function of surface topography, we dipped preweighed, 26.24mm-diameter discs from PDMS replicasts of different plant species (spinach and lettuce), leaf axes (abaxial and adaxial), and leaf ages $(15,45$, and 75 days old, corresponding to $\mathrm{BBCH}$ values of 11,33 , and 49 , respectively) in $50 \mathrm{ml}$ of Milli-Q water for $3 \mathrm{~s}$. Discs were then weighed again to estimate the amount of water that was retained on the surface. In each one of three replicate experiments, we used discs from three different sets of leaves per treatment (plants species, leaf axis, and leaf age), with each set containing four identical PDMS leaf replicasts. Discs of PDMS with flat topography were used as controls. The average volume of water from three replicated experiments was plotted as a function of the leaf surface

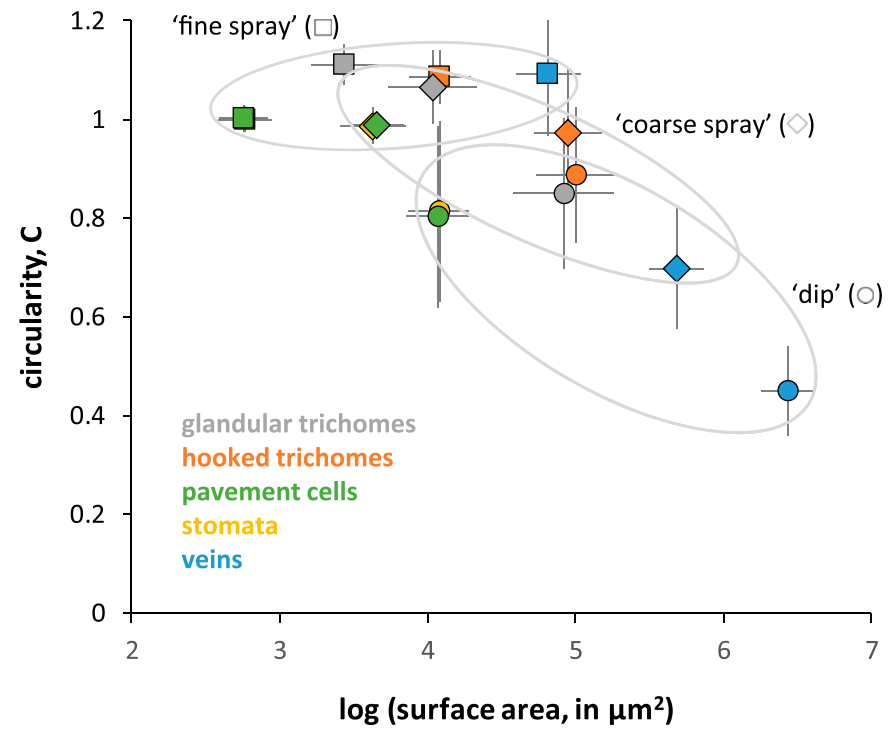

Fig. 2. Relationship between area and shape of individual bodies of water on fresh leaf surfaces as a function of surface structures and application method. For this experiment, bean leaves were wetted with water by dipping, coarse spray, or fine spray, then used as templates in the polydimethylsiloxane (PDMS) casting protocol (Supplementary Fig. S1). Resulting replicasts were analyzed under the microscope to assess the waterscape. Associated with each one of the five major types of leaf structures (i.e., veins, hooked trichomes, glandular trichomes, stomata, and pavement cells), we identified 180 individual bodies of water across three replicasts of three leaves each and measured for each its surface area $\mathrm{A}$ (in square micrometers) and circularity $\mathrm{C}$, which was calculated as the square root of $4 \pi A / P^{2}$, where $P$ is the perimeter. Shown are the averages and standard deviations for those 180 bodies of water for each of the five leaf structures and each of the three wetting methods. Some values for $C$ were greater than 1 due to overestimation by Image $J$ perimeters for round objects from image grids of square pixels. topography of those same discs, measured by microscopy as the percentage of disc surface that was occupied by leaf veins.

To determine the effect of leaf topography on bacterial surface retention, we repeated the water retention experiment as described above but with bacterial cells (Ec70078) or spores (BaFZB42) added to the water at a concentration of $10^{6}$ cells or spores per milliliter. After they were dipped for $3 \mathrm{~s}$ in these bacterial suspensions, discs were transferred into $5 \mathrm{ml}$ of sterile phosphate buffer solution (PBS) (10 mM, pH 7.2) amended with $0.2 \%$ Tween 20 (Thermo Fisher Scientific, Waltham, MA, U.S.A.). Leaf discs were vortexed for $30 \mathrm{~s}$, then sonicated in a Branson sonicator bath (Branson Ultrasonics Corp, St. Louis, MO, U.S.A.) at $250 \mathrm{~W}$ (frequency $=40 \mathrm{kHz}$ ) for $5 \mathrm{~min}$, and vortexed briefly for another 15 s. Surface washes were dilution plated in triplicate on LB (Ec70078) or 0.1× TSA (BaFZB42) using an Eddy Jet spiral plater (IUL, Barcelona, Spain). CFU counts were ${ }^{10} \log$-transformed and averaged for three repeated experiments, then plotted as a function of leaf surface topography of the discs, measured by microscopy as the percentage of disc surface that was occupied by leaf veins.

Bacterial growth on PDMS leaf replicasts. Strain Pa299R:: JBA28 was resuspended in M9 supplemented with kanamycin and rifampin, with or without $0.4 \%$ glucose, to an $\mathrm{OD}_{600}$ of 0.005 (approximately $10^{6}$ bacteria/ml). In a standard experiment (which was performed in triplicate), six PDMS replicasts per surface type (abaxial fresh leaf, PDMS replicast of abaxial, and PDMS flat), nutrient condition (with or without glucose), and time point $(0,6$, $12,18,24,30$, and $36 \mathrm{~h}$ ) were sprayed with approximately $100 \mu \mathrm{l}$ of bacterial suspension with a spray bottle (coarse) from a distance of $30 \mathrm{~cm}$. To achieve different levels of relative humidity (RH), inoculated surfaces were placed in 100-mm-diameter plastic Petri dishes at $23^{\circ} \mathrm{C}$ as follows: (i) on top of water-saturated germination paper (catalog number CDB 3 3/8" Circle; Anchor Paper Co., St. Paul, MN, U.S.A.), after which the Petri dish was sealed with parafilm (= high RH); (ii) on top of a water-saturated germination paper but the dish was not Parafilmed (= medium RH); or (iii) on top of four layers of paper towels (Koch Industries, Wichita, KS, U.S.A.), after which the Petri dish was taped closed with parafilm (= low RH). At each sampling time point $(0,6,12,18,24,30$, and $36 \mathrm{~h}$ after inoculation), six leaf replicasts per surface type, nutrient condition, and level of RH were individually transferred to a 50-ml Falcon tube (VWR International) containing $20 \mathrm{ml}$ of PBS (10 mM, $\mathrm{pH}$ 7.2), vortexed for $15 \mathrm{~s}$, sonicated for $7 \mathrm{~min}$ in a Branson 5510 sonicator bath (Emerson Electric Company), and vortexed for $15 \mathrm{~s}$ again. Diluted aliquots from each surface wash were plated in triplicate onto LB plates containing kanamycin and rifampicin using an Eddy Jet spiral plater (IUL). CFU counts (CFU + 1) were normalized per square centimeter of total surface area, as determined by ImageJ software, then ${ }^{10} \log$-transformed and averaged among all six PDMS replicasts per surface type, time point, level of $\mathrm{RH}$, and nutrient condition. For these mean population sizes, the average and standard deviation for each time point was calculated and plotted as a function of surface type, level of $\mathrm{RH}$, and nutrient condition.

Statistics. Statistical analysis of the data were performed in Microsoft Excel or using R version 3.3.2 (The R Foundation for Statistical Computing, Vienna, Austria). Unless otherwise indicated, data were analyzed using a one-way analysis of variance with posthoc Tukey's honestly significant difference.

\section{RESULTS}

Fabrication of topomimetic leaf surfaces. Leaf surface topographies of green snap bean plants were successfully replicated using PDMS in a two-step casting protocol (Supplementary Fig. 
S1). SEM of fresh bean leaves and their PDMS replicasts revealed faithful, high-resolution reproductions of various leaf structures, including veins, epidermal pavement cells, trichomes, and stomata (Fig. 1A to H). Fresh leaves (Fig. 1A to D) more than PDMS replicasts (Fig. 1E to $\mathrm{H}$ ) showed artifacts such as surface shrinkage and distortion, which are typical of SEM sample preparation (Pathan et al. 2010). Our casting process also worked well with leaves from plants other than beans representing a wide range of surface topographies, including potato (Fig. 1I), zebra plant (Fig. 1J), tomato (Fig. 1K), and lettuce (Fig. 1L).

The PDMS replicasts have several features that make them highly suitable for experimentation related to the study of microbial colonization of the phyllosphere. First, they are compatible with a wide range of microscopy techniques, including light, fluorescence, electron, and laser microscopy (Supplementary Fig. $\mathrm{S} 3$ ). Second, the PDMS replicasts proved to be highly tolerant of various sterilization and decontamination practices that permit gnotobiotic conditions and the reuse of replicasts; SEM imaging confirmed that surface features were preserved following exposure to bleach or phosphoric acid, or after autoclaving (Supplementary Fig. S4). Third, our protocol permitted the manufacture of up to four identical PDMS replicasts from a single negative mold without degradation of topographical quality (Supplementary Fig. S5A and B). Key to success for this was the coating of the negative PDMS mold with a monolayer of an organic compound, octadecyltrichlorosilane (ODTS), which renders the mold surface hydrophobic, prior to using the mold to produce positive replicasts (Supplementary Fig. S1C and D) (Materials and Methods) (Parikh et al. 1994). Without this coating step (Supplementary Fig. S5C and D), artifacts were introduced into the PDMS replicasts by the second time the same negative mold was used (Supplementary Fig. S5D), most likely as a result of microtearing of the noncoated

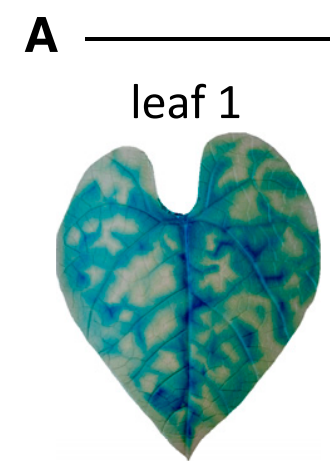

$4.36^{a}$

(448)

\section{abaxial}

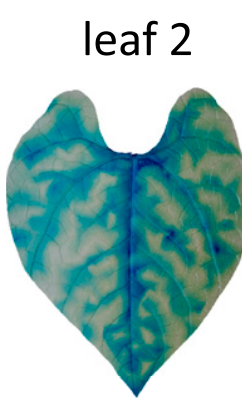

$4.02^{b}$

(465)

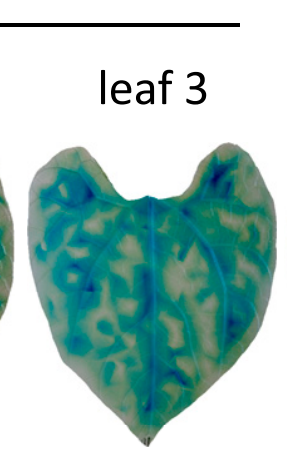

$3.36^{c}$

(303)

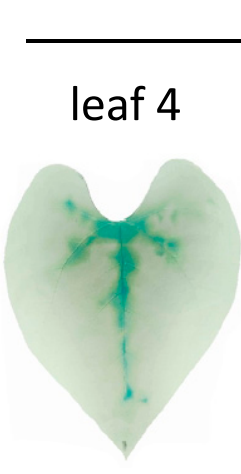

$1.61^{\mathrm{d}}$

(362)

\section{adaxial leaf 5}

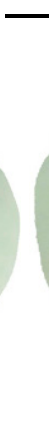

$1.69^{d}$

(527)

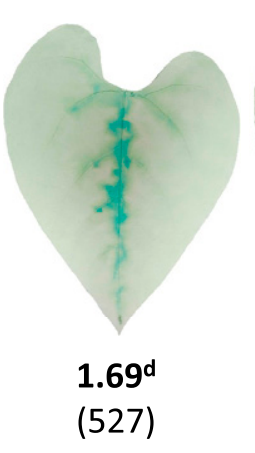

leaf 6

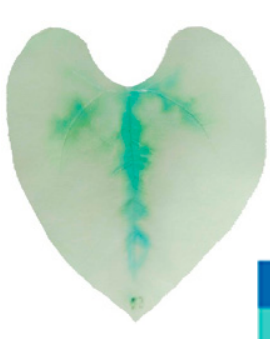

$6.5 \mathrm{~cm}$

$4.5 \mathrm{~cm}$

$1.60^{\mathrm{d}}$

$2.5 \mathrm{~cm}$

(418)

$0.5 \mathrm{~cm}$

B

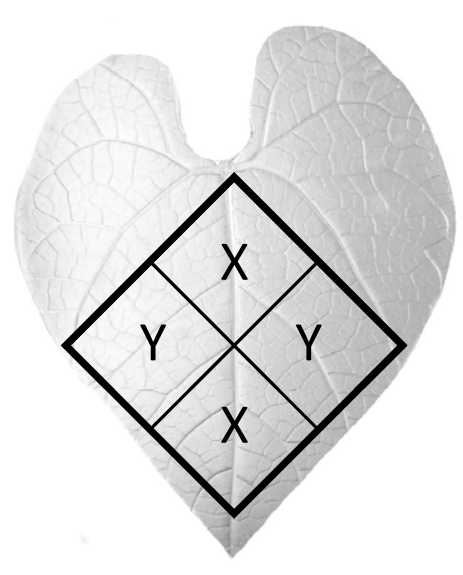

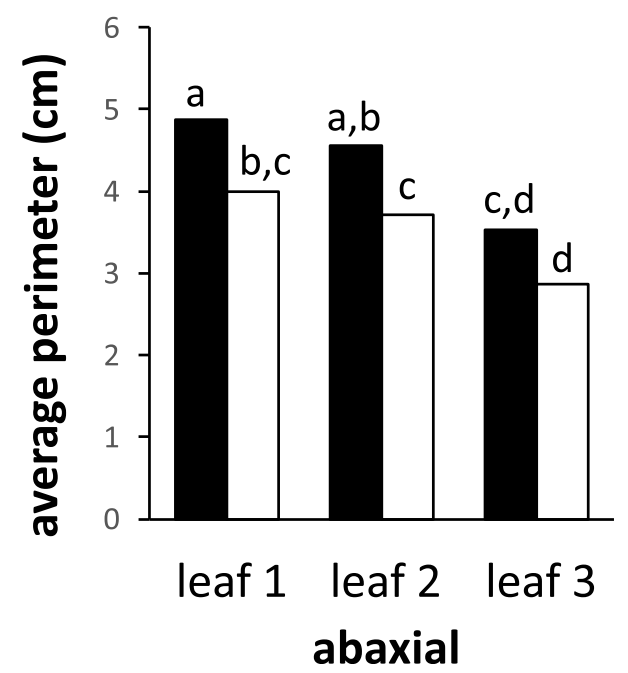

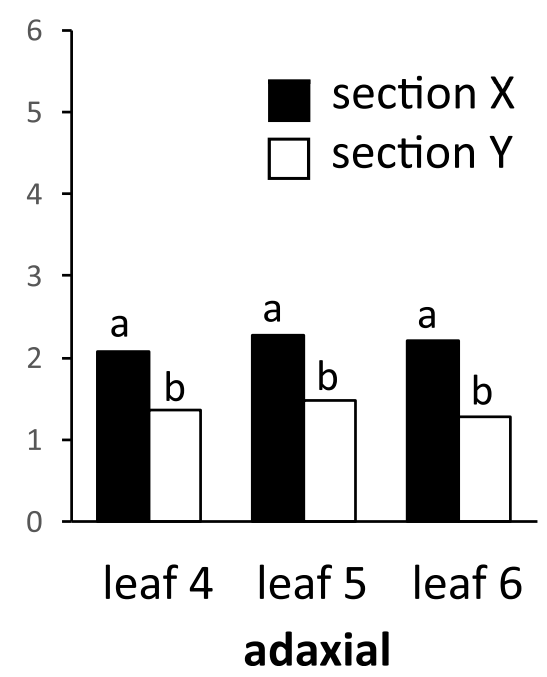

Fig. 3. Surface spread of water as a function of leaf axis and topography. For this experiment, water drops ( $1 \mu$ l each, containing $0.0005 \%$ brilliant cresyl blue and $0.015 \%$ Silwet) were placed at arbitrary locations on polydimethylsiloxane (PDMS) replicasts of the abaxial or adaxial side of bean leaves. Using ImageJ image analysis, we measured the perimeter (in centimeters) and x,y location of each drop. A, Shown are heatmaps generated with GIS version 2.18.3 using the drop data combined from 12 identical PDMS replicasts for each one of six bean leaf surfaces (three abaxial, leaves 1 to 3 ; and three adaxial, leaves 4 to 6 ). For each one of the six leaves, the average drop perimeter is given; values with the same superscript letter were not significantly different from each other. Shown in parentheses are the number of drops analyzed per leaf. In the heat maps, areas of the PDMS surface that allowed greater spread of the water drop appear darker blue. The color legend gives gradations of blue for four perimeter values $(0.5,2.5,4.5$, and $6.5 \mathrm{~cm}$ ); the largest perimeter measured was close to $7 \mathrm{~cm}$. B, For each one of the six leaves $(1$ to 6$)$, the perimeters of all drops in quadrants labeled $X$ were averaged, as were the perimeters of all drops in quadrants labeled $Y$. Shown are the six averages for leaves 1 to 3 as well as the six averages for leaves 4 to 6 . Abaxial averages with the same lettering were not significantly different $(P<0.01)$ from each other. Adaxial averages with the same lettering were not significantly different $(P<0.01)$ from each other. 
negative mold during separation from the first positive cast (Supplementary Fig. S1B).

Quantifying the shape of the leaf surface waterscape using PDMS replicasts. By applying our PDMS casting protocol to wet leaf surfaces, we were able to generate by SEM a realistic and quantifiable reconstruction of the shape of the leaf surface waterscape in relation to topography. Different waterscapes were produced depending on the way in which the leaves were wetted and on the local topography of the leaf surface. A brief immersion (dip) of leaves in water resulted in bodies of water that were large and contiguous (Supplementary Fig. S6A), whereas coarse and fine water sprays resulted in more fragmented waterscapes typically made up of medium and small bodies of water, respectively (Supplementary Fig. S6B and C). A quantitative analysis of the SEM images revealed that, in terms of size (expressed as surface area), water bodies were always the smallest near pavement cells or stomata and the largest near veins, independent of the wetting method (Fig. 2). Across the same leaf structure, however, the size of water bodies was typically the largest with the dip method, followed by coarse spray, then fine spray (Fig. 2). In terms of shape (expressed as circularity $\mathrm{C}$, where 1 is the maximum value and represents a perfect circle), round droplets of water were most commonly observed, as expected, with the two spray methods (coarse and fine) in association with pavement cells, stomata, and the two types of trichomes (hooked and glandular). The largest deviation from the circular shape was observed near veins when using the dip and coarse spray methods (Fig. 2). This finding is consistent with the SEM images, which often showed oblong bodies of water paralleling leaf veins (Supplementary Fig. S6A and B). We interpret these results to mean that certain leaf structures, in particular leaf veins, facilitate the coalescence of discrete water droplets into larger, contiguous bodies of water.

Shape and spread of surface water on topomimetic leaves. Compared with fresh bean leaf surfaces, PDMS replicasts of the same bean leaf surfaces were found to hold water drops with a significantly larger contact angle (Supplementary Table S1). This is consistent with the hydrophobic nature of PDMS (Sun et al. 2019). The addition of $0.2 \%$ (vol/vol) Silwet L77 to the water reduced contact angles to levels that were comparable with, and not significantly different from, those observed for fresh bean leaves (Supplementary Table S1). There were no significant differences in contact angles between adaxial and abaxial surfaces or between topomimetic and flat PDMS surfaces (Supplementary Table S1). Based on these results, and unless otherwise noted, we supplemented water that was introduced to PDMS replicasts with $0.2 \%$ Silwet.
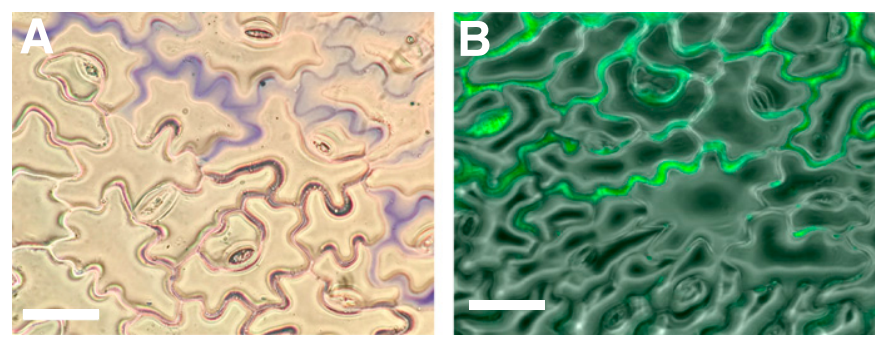

Fig. 4. Leaf surface topography-driven spread of water at the microscopic scale. Water drops containing either $\mathbf{A}, 0.0005 \%$ brilliant cresyl blue or B, green fluorescently labeled Pantoea agglomerans 299R::JBA28 cells were spotted onto polydimethylsiloxane (PDMS) replicasts and examined by light $(A)$ or fluorescence $(B)$ microscopy. Images $A$ and $B$ do not represent the same field of view. The bar in both panels represents $50 \mu \mathrm{m}$.
To quantify the topography-dependent spread of water on leaf surfaces, we spot-inoculated PDMS replicasts of adaxial and abaxial bean leaf surfaces, as well as flat PDMS surfaces, with 1- $\mu$ l drops of a suspension of brilliant cresyl blue in water. This dye allowed visualization of the spread of individual water drops on leaves, whether fresh or PDMS replicasts (Supplementary Fig. S7). Quantitative analysis revealed that this spread (expressed as the perimeter of the drop's footprint on the surface) was significantly greater on the abaxial surface, which features more pronounced leaf venation, than on the adaxial surface (Fig. 3A). Also, the degree of spread appeared greatest in areas of the leaf with primary and, to a lesser extent, secondary veins (Fig. 3A). Across six different leaf surfaces, average spread was significantly greater in areas that encompassed primary veins than in areas that did not, for all three adaxial leaf surfaces and for two of the three abaxial leaf surfaces (Fig. 3B). On flat PDMS surfaces, the spread of individual water drops was minimal (not shown).

The path by which water spread away from major leaf veins appeared to be dictated by fine-scale leaf surface topography, as shown by microscopy (Fig. 4). More specifically, water to which brilliant cresyl blue (Fig. 4A) or green fluorescently labeled bacteria (Fig. 4B) was added was seen to spread through the gorges in between pavements cells. These results also suggest that the topography-driven lateral spread of water on leaf surfaces contributes to the lateral spread of bacteria on leaf surfaces.

Impact of the interaction between leaf topography and surface water on bacterial spheres of perception in the phyllosphere. Given the ability of leaf veins to act as conduits for leaf surface water (Fig. 3A), we used our PDMS replicasts to ask how the topography-dependent waterscape on leaves influences the distances along which bacteria can sense their environment. For this experiment, IPTG-responsive bacteria were tested for their ability to sense IPTG over increasing distances (on a millimeter scale) either along or perpendicular to secondary veins on PDMS replicasts of abaxial bean leaf surfaces (Fig. 5). When bacteria and IPTG were inoculated on the same side of a vein, bacteria were able to sense IPTG from a distance as far as $24 \mathrm{~mm}$ away but only when IPTG and bacteria were both spotted right next to the vein (Fig. 5A). The ability of bacteria to sense IPTG diminished when IPTG or bacteria were located further away from the vein (Fig. 5B to D). For IPTG that was dropped right next to a vein (Fig. 5A), the incidence of sensing beyond a distance of $2 \mathrm{~mm}$ was significantly higher for bacteria along the vein compared with bacteria away from the vein (Fig. 5E). Also, there was a significantly higher incidence of bacterial sensing beyond a parallel-to-the-vein distance of $2 \mathrm{~mm}$ (Fig. 5F) if IPTG was placed $0 \mathrm{~mm}$ away from the vein (Fig. 5A) compared with $8 \mathrm{~mm}$ away from the vein (Fig. 5D). This finding speaks to the function of veins as conduits of water and any chemicals contained within that water. When bacteria and IPTG were placed on opposite sides of secondary vein, bacteria never were able to sense the IPTG (data not shown). On flat surfaces, bacteria were not able to sense IPTG even from a distance of $2 \mathrm{~mm}$ (data not shown).

Retention of water and bacteria on PDMS replicast surfaces with different topographies. To assess whether leaf surface topography had an effect on not only the shape and spread but also retention of water, we measured the amount of liquid that remained attached to circular sections (i.e., discs, with a diameter of $24 \mathrm{~mm}$ ) of PDMS replicasts of lettuce and spinach leaves after dipping in water. This assessment revealed a significant correlation between surface topography, measured as percentage of leaf surface area that is covered by veins, and surface water retention (Fig. 6A). For both lettuce and spinach leaves, abaxial surfaces had more venation and retained more water than adaxial surfaces (Fig. 6B). Spinach leaves of different ages did not show significant differences in surface area 


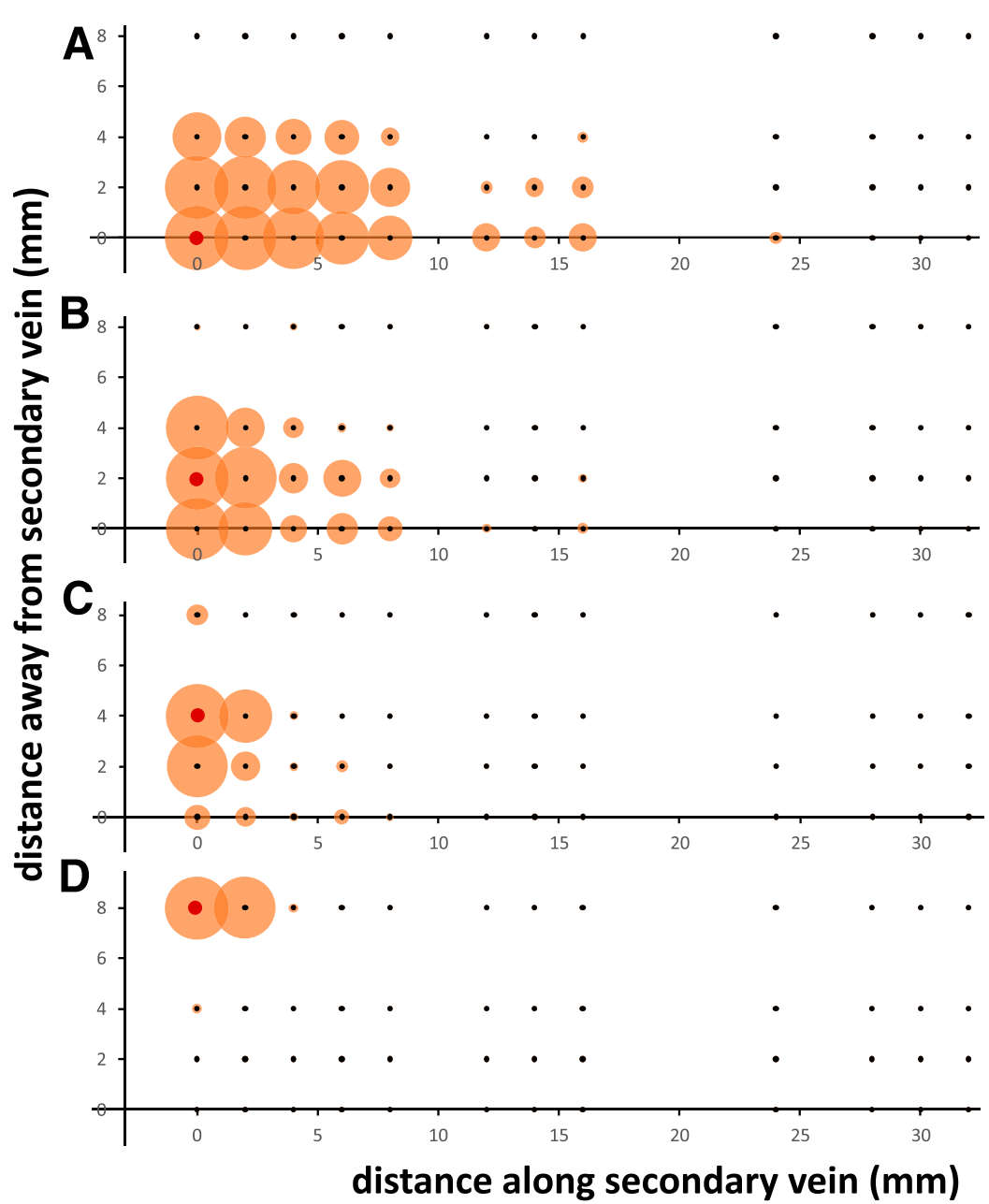

$\mathbf{E}$

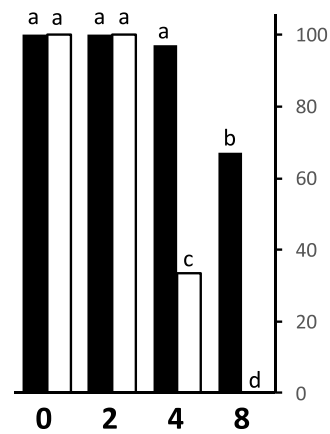

distance along (black bars) or away (white bars) from the vein $(\mathrm{mm})$

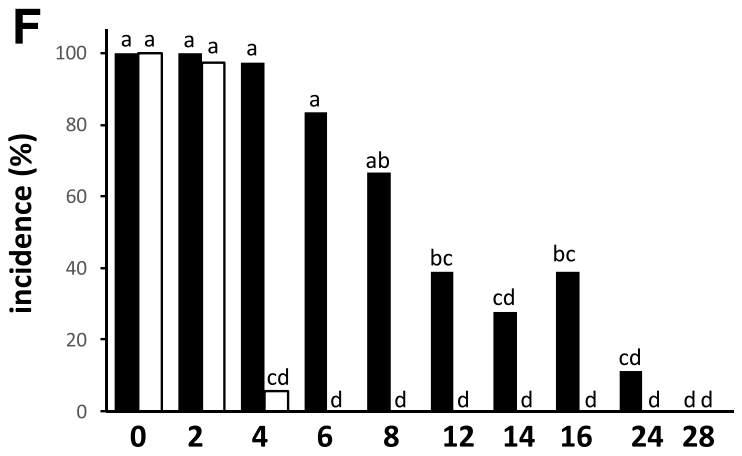

distance parallel to the vein $(\mathrm{mm})$, with IPTG placed at 0 (black bars) or 8 (white bars) $\mathrm{mm}$ away from the vein

Fig. 5. Scatter plot documenting the ability of isopropyl- $\beta$-D-thiogalactopyranosid (IPTG)-responsive bacterium Pantoea agglomerans $299 \mathrm{R}::$ JBA28 (pCPP39) to sense IPTG along various distances along and away from secondary veins on polydimethylsiloxane (PDMS) replicasts of the abaxial side of bean leaves. Red dots indicate locations where drops of concentrated IPTG were spotted A, right next to a secondary vein (0 $\mathrm{mm}$ ) or B, $2 \mathrm{~mm}$; C, $4 \mathrm{~mm}$; or D, $8 \mathrm{~mm}$ away from a secondary vein. Black dots represent locations where IPTG-responsive bacteria were spot inoculated. Each black dot represents up to 54 replications across multiple secondary veins, multiple PDMS replicasts, and multiple repeats $(n=3)$ of the experiment (for a detailed description of the experimental design, see Materials and Methods and Supplementary Figure S2). Each one of those replications was scored as positive (i.e., green fluorescent protein [GFP] was detected, as determined by fluorescence microscopy) or negative (i.e., no GFP was detected). The size of the orange halo around each black dot is proportional to the incidence (i.e., percentage of replications that scored positive). As a positive control, we spotted bacteria on top of the spotted IPTG (red dots); these all scored $100 \%$. E, Incidence as a function of distance along the vein or away from the vein. For this analysis, we calculated for each one of the triplicate experiments the incidence values as a function of distance $(0,2,4$, or $8 \mathrm{~mm})$ from an IPTG spot placed right next to a vein, either along the vein (black bars) or away from the vein (white bars). Shown are the averages of these incidence values. Incidence values with the same letter are not significantly different $(P<0.01)$ from each other. $\mathbf{F}$, Incidence as a function of distance parallel to the vein, either right next to the vein or at a greater distance. For this analysis, we calculated for each one of the triplicate experiments the incidence values as a function of parallel-to-the-vein distance $(0,2,4,6,8,12,14,16,24,28,30$, or $32 \mathrm{~mm})$ from an IPTG spot that was placed either at a distance of $0 \mathrm{~mm}$ from the vein (as in A; black bars in this panel) or $8 \mathrm{~mm}$ from the vein (as in D; white bars in this panel). Shown are the averages of these incidence values. Incidence values with the same letter are not significantly different $(P<0.01)$ from each other. 


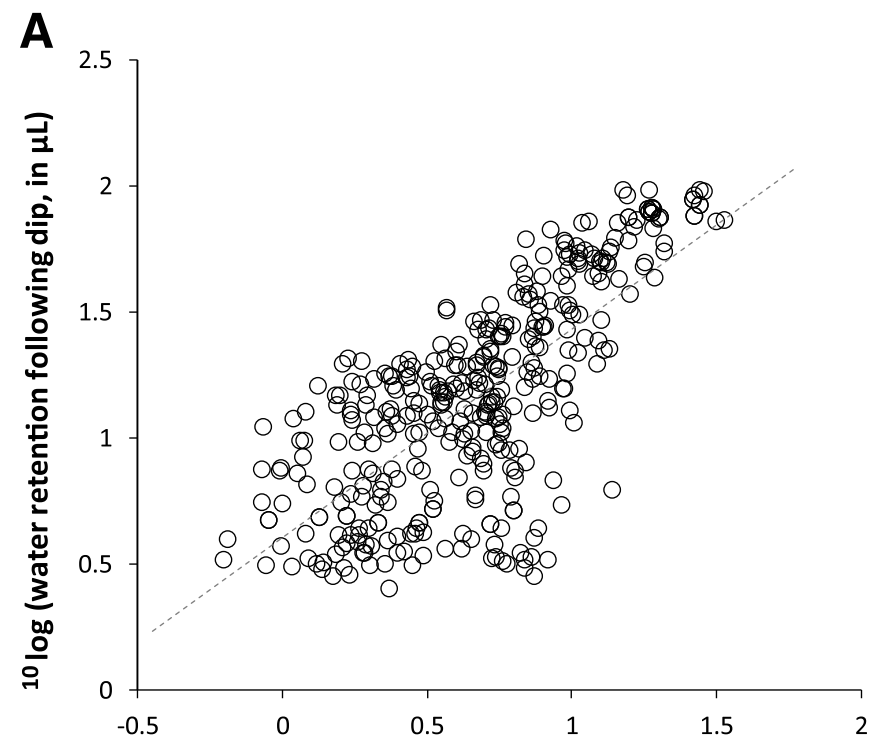

B

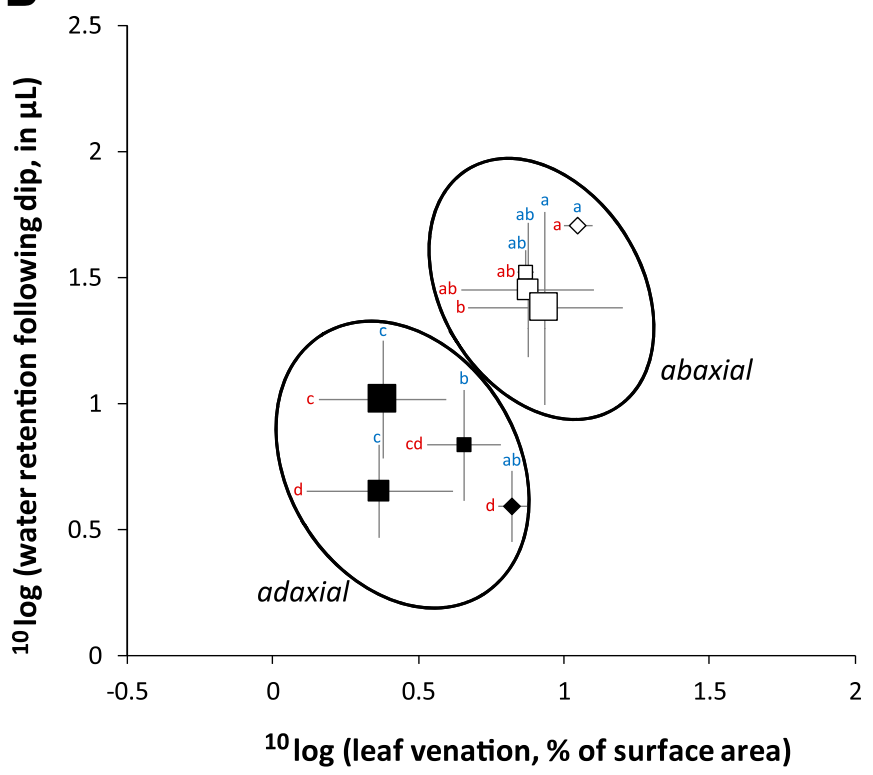

Fig. 6. Surface water retention as a function of leaf venation, axis, and age. A, For 366 individual discs from polydimethylsiloxane (PDMS) replicasts of lettuce and spinach leaves, the volumes of water (in microliters) that were retained on the surface after brief immersion in water ( $y$-axis) as a function of the percentage of leaf surface covered by veins ( $x$-axis). The stippled trendline highlights the positive relationship between leaf venation and water retention (goodness of fit $R^{2}=0.5087$; slope significantly nonzero: $P<0.0001$ ). B, Data averaged over leaf axis, leaf age, and plant species: diamonds represent young $(\mathrm{BBCH}=11)$ lettuce leaves, squares represent spinach leaves (small, medium, and large squares correspond to $\mathrm{BBCH}$ values of 11,33 , and 49 , respectively), filled symbols represent adaxial surfaces, and open symbols represent abaxial surfaces. Error bars represent standard deviations. Data points that are labeled with the same blue letter are not significantly different $(P<0.01)$ from each other in terms of leaf venation. Data points that are labeled with the same red letter are not significantly different $(P<0.01)$ from each other in terms of water retention.

or water retention on their abaxial side (Fig. 6B). The adaxial side of young leaves $(\mathrm{BBCH}=11)$ showed, on average, more venation than that of older leaves $(\mathrm{BBCH}=33$ or 49$)$ but not more water retention (Fig. 6B). Interestingly, the degree of venation of adaxial lettuce leaves and abaxial spinach leaves was not significantly different but adaxial lettuce leaves retained significantly less water than abaxial spinach leaves (Fig. 6B). We infer from this that vein surface area alone is not a perfect predictor of water retention and that the shape of the vein, or more specifically the degree or ribbing (i.e., the protrusion of [especially primary] veins from the surface, which is much greater on abaxial spinach leaves than on adaxial spinach leaves), plays an important role in the retention of water. The typical volume of water retained by flat PDMS discs, which completely lack venation or any other topography, was 1 to $2 \mu \mathrm{l}$ (data not shown).

To quantify the water-assisted attachment of bacteria to leaves with different topographies, we performed a variation on the water retention experiment in which PDMS replicasts were briefly dipped into a suspension of $10^{6}$ bacterial cells $(E$. coli) or spores (B. amyloliquefaciens) per milliliter, after which the number of bacteria that were retained on the surface was estimated by spread plating of PDMS surface washes. As expected, more bacteria were recovered from PDMS replicasts of abaxial than of adaxial leaf surfaces (Fig. 7). The largest difference between abaxial and adaxial retention on leaves of the same type was found for lettuce; abaxial surfaces of lettuce leaves retained, on average, approximately 12 times more bacteria than adaxial surfaces of the same lettuce leaves. No significant difference in retention was observed between $E$. coli cells (Fig. 7A) and B. amyloliquefaciens spores (Fig. 7B), suggesting that their retention is driven more by the interaction of the water with the leaf topography than by differences in surface properties or size between bacterial cells and bacterial spores. Very few bacterial cells and spores were retained on surfaces without topography (i.e., flat PDMS) (data not shown).

Bacterial growth and survival on PDMS replicasts of leaf surfaces. To assess the effect of leaf topography on bacterial colonization and survival, we used a coarse-spray inoculation protocol to deliver a strain of the bacterial phyllosphere colonizer P. agglomerans onto the abaxial surface of detached fresh bean leaves, onto PDMS replicasts of the abaxial surface of bean leaves, or onto flat PDMS, with or without glucose as a carbon source, and under three different levels of RH (see Materials and Methods section for operational definitions of these relative levels of RH). Changes in bacterial population sizes were determined by spread plating of surface washes at 6-h intervals up to $36 \mathrm{~h}$ (Fig. 8). In the absence of added glucose (Fig. 8, dashed lines), a significant increase in bacterial population size was observed only on fresh leaf surfaces (Fig. 8, circles) and only under conditions of high and medium RH (Fig. 8A and B). At low $\mathrm{RH}$, conditions on these fresh leaves were not conducive to bacterial growth or survival, even in the presence of added glucose (solid lines). Independent of glucose, bacterial population sizes declined at an estimated rate of $-0.13 \mathrm{~h}^{-1}$ following inoculation (Fig. 8C). On PDMS replicasts (Fig. 8, triangles), significant bacterial growth was observed only with the addition of glucose, as expected (i.e., the PDMS is not a source of carbon), but only under conditions of high and medium RH (Fig. 8A and B). The increase in population size in response to added glucose at high RH was not significantly different between topomimetic or flat PDMS leaves or between PDMS replicasts and fresh leaves (Fig. 8A). The glucose-induced 100-fold higher population sizes on fresh leaves at high and medium RH are consistent with the notion that bacterial growth in the phyllosphere is nutrient limited (Lindow and Brandl 2003). Without added glucose, the bacterial population size on both topomimetic and flat PDMS surfaces declined more rapidly with decreasing RH levels (Fig. 8A to C). However, at low RH (Fig. 8C), the decline was significantly greater on flat PDMS surfaces than on topomimetic PDMS replicasts. More specifically, the death rate was 2.4- or 1.8-fold higher on flat PDMS in the absence or presence of glucose, respectively. 


\section{DISCUSSION}

We reported here on a protocol for replica casting (replicasting) fresh leaf surfaces into elastomeric PDMS to yield microscopic topographies that offer wide experimental utility in the field of phyllosphere microbiology. Our protocol differs in one or more ways from those previously published for this purpose. First, it allowed us to produce multiple positive replicasts from a single negative mold, which is an asset in experiments that need to control for leaf surface topography in order to test the effect of other factors, including chance events (Maignien et al. 2014). Our protocol allowed the four-time use of a single negative mold, treated one time with ODTS, to produce leaf surface replicasts; however, this may be improved upon by treating the negative mold after every use with trichloro(1H,1H,2H,2H-perfluorooctyl)silane (Zhang et al. 2010). Second, mold curing was done at lower temperatures $\left(30\right.$ to $\left.33^{\circ} \mathrm{C}\right)$ or for a longer period of time $(17 \mathrm{~h})$ compared with other studies (Soffe et al. 2019b; Zhang et al. 2014). Here, we exploited this protocol to demonstrate that three different methods of leaf inoculation commonly used in phyllosphere microbiology (leaf dip, coarse spray, and fine spray) yielded quantitatively very different waterscapes. Such differences are likely to influence the interactions that the introduced microorganisms will have with the plant host, as several studies show. For example, E. coli survived better on lettuce leaves when sprayed from a bottle compared with spot inoculation (Moyne et al. 2013). A spore suspension of Alternaria brassicae onto the leaves of Indian mustard led to more severe symptoms when deposited by pipetting drops than by spraying (Giri et al. 2013). Similar differences were reported for drop-versus-spray inoculations of bean leaves with spores of the pathogenic fungus Elsinoe phaseoli (Phillips 1994). Together with our findings, these observations suggest that caution is justified when comparing data from phyllosphere colonization studies that use different inoculation techniques.

The ability to generate PDMS replicasts from wetted fresh leaves (Supplementary Fig. S6) presented an opportunity to quantitatively describe a role for leaf surface topography in shaping the phyllotelma. Veins in particular, and to a lesser degree trichomes, collected larger bodies of water than other surface features such as stomata and pavement cells (Fig. 2). Leaf veins and trichomes also are known as sites that phyllosphere bacteria such as Pseudomonas syringae associate with more abundantly than other parts on the leaf (Monier and Lindow 2004). It is generally assumed that this bacterial preference for veins and trichomes is linked to a higher availability of nutrients at these sites compared with elsewhere (Beattie 2011; Monier and Lindow 2004). Although there are studies that support such a premise (Remus-Emsermann et al. 2011), our findings here are consistent with an alternative, not mutually exclusive, explanation that the accumulation of bacteria near veins and trichomes is an outcome of the interaction of water (and any particles it may hold, including bacteria) with a laterally heterogeneous surface topography. From this, it follows that the microbial distribution on leaf surfaces may, to a certain degree, be predicted from the behavior of water along the V-shaped crevices along protruding veins and in valleys between epidermal cells. The physics of capillary flow in open channels are generally well understood (Tian et al. 2010; Yang et al. 2011) and have been successfully applied toward improving surface area coverage of leaves and foliage with agrochemicals (Januszkiewicz et al. 2019; Taylor 2011). The quantitative study of leaf surface water flow dynamics is relatively underexplored in phyllosphere microbiology (Grinberg et al. 2019) but holds promise to explain and predict microscopic variations in microbial community structure, the success or failure of foliarly applied biocontrol agents, and the efficacy of washing protocols designed to remove bacterial contaminants from leafy greens.

Veins spread water significantly better than other parts of the leaf surface (Fig. 3) and contribute considerably to the retention of water on briefly submerged leaves (Figs. 6 and 7). Veins also have been described as sites on the leaf surface which, under conditions of low
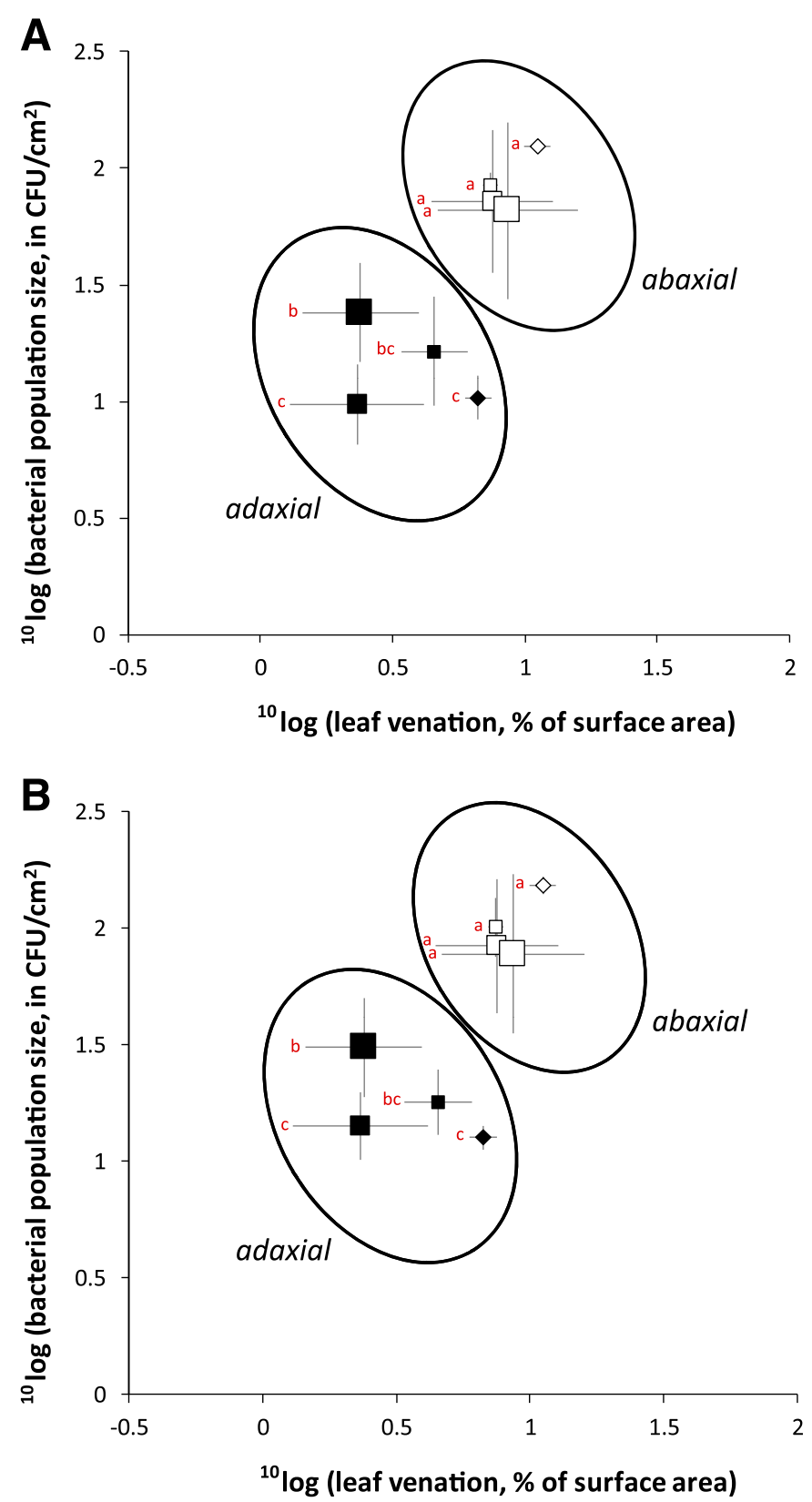

Fig. 7. Retention of bacterial cells or spores as a function of leaf venation, axis, and age. Shown are the average number of CFU that were retained on the surface of discs from polydimethylsiloxane (PDMS) replicasts of lettuce and spinach leaves after brief immersion in a suspension of A, bacterial cells (Escherichia coli ATCC 700728) or B, spores (Bacillus amyloliquefaciens FZB42), as a function of the percentage of leaf surface covered by veins. In both panels, diamonds represent young $(\mathrm{BBCH}=11)$ lettuce leaves, squares represent spinach leaves (small, medium, and large squares correspond to $\mathrm{BBCH}$ values of 11,33 , and 49 , respectively), filled symbols represent adaxial surfaces, and open symbols represent abaxial surfaces. Error bars represent standard deviations. Data points that are labeled with the same red letter are not significantly different $(P<0.01)$ from each other in terms of bacterial population size. 

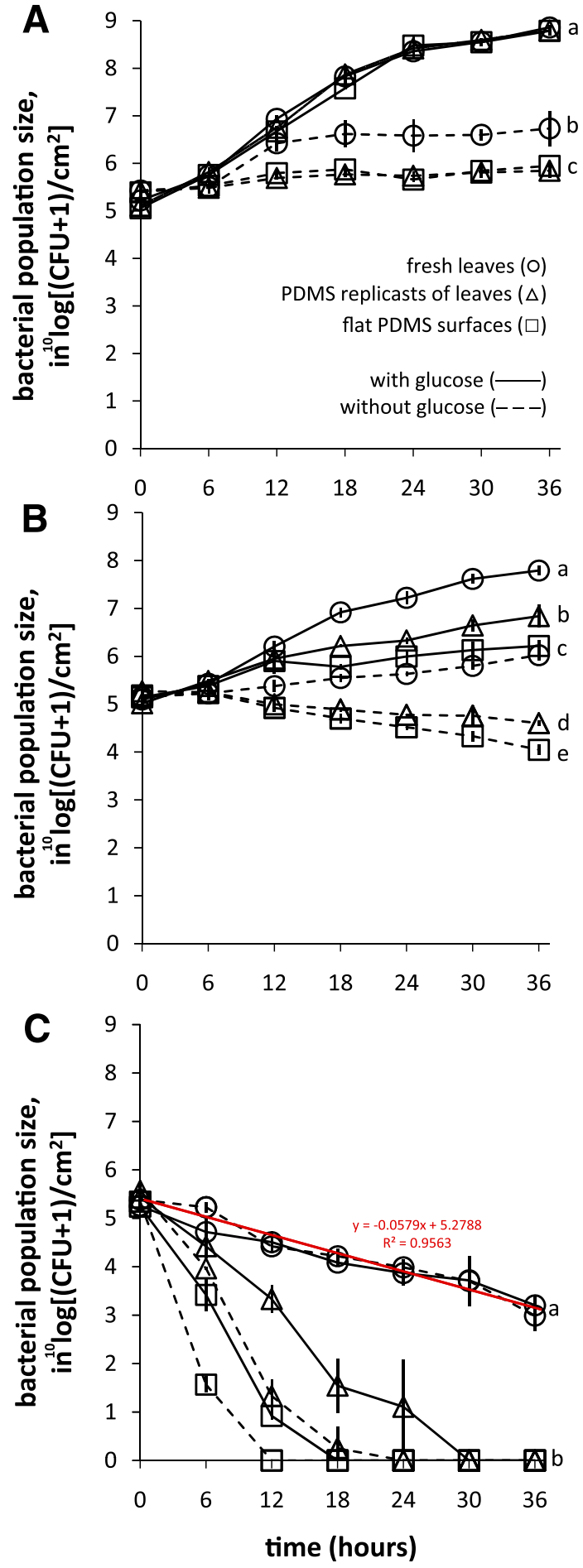

Fig. 8. Bacterial population dynamics on fresh leaves and polydimethylsiloxane (PDMS) replicasts. Shown are the population sizes of Pantoea agglomerans 299R::JBA28 as a function of time after coarse-spray inoculation onto fresh bean leaves (circles), PDMS replicasts of bean leaves (triangles), or flat PDMS surfaces (squares), in the presence (solid lines) or absence (dashed lines) of $0.4 \%$ glucose, and incubated under conditions of $\mathbf{A}$, high; $\mathbf{B}$, medium; or $\mathbf{C}$, low relative humidity $(\mathrm{RH})$. Average and standard deviation were calculated for six replicates per surface type, glucose condition, $\mathrm{RH}$ level, and time point. In each panel, $\mathrm{t}=36$-h population sizes that are labeled with the same letter are not significantly different $(P<0.01)$ from each other. In $\mathrm{C}$, the rate of decline for bacterial population sizes on fresh leaves, with and without added glucose, was calculated by dividing the slope of the (red) fitted regression line $(-0.0579)$ by $\log (e)$.
$\mathrm{RH}$, retain water longer than other leaf surfaces structures (Brewer et al. 1991) and where bacteria are less likely to experience water stress than in sites away from veins (Axtell and Beattie 2002). Thus, veins offer phyllosphere microorganisms protection from desiccation, which is part of the explanation for the higher observed population sizes of viable bacteria on abaxial compared with adaxial leaf surfaces (Leben 1988). This invites the question of whether leaf venation, or leaf topography in general, may be manipulated to control the numbers or types of microorganisms, in particular those that are unwanted such as foliar pathogens. One option is selective plant breeding, which takes advantage of the fact that leaf surface topography is a genetic and heritable trait and is being explored to reduce leaf surface roughness and unpalatability of grasses for forage (Hartley et al. 2015). Another option is the application of foliar films that disguise cues necessary for pathogen establishment (Walters 2006). Although some of these cues are chemical, others are physical and directly related to leaf surface topography. Both options (breeding and foliar application) stand to gain from PDMS replicasts as a research tool, particularly if there is a need to separate the effects of topography from other factors such as plant metabolites and cuticle chemistry.

Seeing the variation in shape and size of water bodies on the leaf surface (Fig. 2) brings to light our limited understanding of how the ecological notion of "habitat fragmentation" (Fahrig 2003) might apply to the phyllosphere at microscopic scales. Consider the bacterial cells that inhabit the single droplet shown in Supplementary Figure S3B. Are these bacteria in their own little ecosystem, interacting only among themselves, affected and supported only by the part of the plant that is covered by the drop of water in which they reside? Such a model of many smaller and unconnected microhabitats may well promote microbial coexistence (Wang and Or 2013) and explain the microbial diversity that is typically associated with leaf surfaces (Schlechter et al. 2019). This model also is in line with the idea that a leaf consists of many independent sites, each one representing a "local carrying capacity" (RemusEmsermann et al. 2012). Alternatively, are drops like the one shown in Supplementary Figure S3B connected and part of larger bodies of water that allow the diffusion and translocation of chemicals and microbes along larger distance along the leaf surfaces? In more general terms, these questions pertain to the concept of overlapping microbial spheres of influence and perception (Leveau 2009; Remus-Emsermann and Schlechter 2018), which supposes that the size, shape, and connectivity of the waterscape facilitates or constrains the ability of microorganisms to communicate with each other, influence or sense each other, or compete for shared resources. Our experiment with IPTG-responsive bacteria (Fig. 5) demonstrates that leaf surface topography plays a critical role in shaping these spheres. One major takeaway from the experiment is that proximity is not a good predictor of interaction. Veins were an insurmountable obstacle for bacteria in sensing IPTG just on the other side of such a vein. However, when bacteria and IPTG were spotted on the same side along a vein, spheres of IPTG influence and bacterial perception overlapped over distances as far as $24 \mathrm{~mm}$ (Fig. 5). By contrast, overlap in spheres of IPTG influence and bacterial perception declined rapidly with increasing perpendicular distances from the vein. This quantitative understanding of waterscape-dependent connectivity is meaningful in the context of interactions between microorganisms of the same species; for example, as it relates to quorum sensing (Dulla and Lindow 2008) or microorganisms representing different species; for example, as it relates to overlap in nutrient utilization patterns (Innerebner et al. 2011; Wilson and Lindow 1994). PDMS replicasts of leaf surfaces are expected to prove extremely useful for quantifying such spheres, given the typically ephemeral nature of the leaf surface waterscape 
(Beattie 2011) and the recently uncovered fact that microorganisms themselves, in the form and shape of bacterial aggregates, represent enough "topography" to retain water and contribute to microscopic patterns of leaf wetness (Grinberg et al. 2019).

\section{LITERATURE CITED}

Axtell, C. A., and Beattie, G. A. 2002. Construction and characterization of a proU-gfp transcriptional fusion that measures water availability in a microbial habitat. Appl. Environ. Microbiol. 68:4604-4612.

Barthlott, W., Mail, M., Bhushan, B., and Koch, K. 2017. Plant surfaces: Structures and functions for biomimetic innovations. Nano-Micro Lett. 9:23.

Beattie, G. A. 2011. Water relations in the interaction of foliar bacterial pathogens with plants. Annu. Rev. Phytopathol. 49:533-555.

Bogino, P. C., Oliva, M. D., Sorroche, F. G., and Giordano, W. 2013. The role of bacterial biofilms and surface components in plant-bacterial associations. Int. J. Mol. Sci. 14:15838-15859.

Bonaterra, A., Cabrefiga, J., Camps, J., and Montesinos, E. 2007. Increasing survival and efficacy of a bacterial biocontrol agent of fire blight of rosaceous plants by means of osmoadaptation. FEMS Microbiol. Ecol. 61: 185-195.

Brandl, M., Clark, E. M., and Lindow, S. E. 1996. Characterization of the indole-3 acetic acid (IAA) biosynthetic pathway in an epiphytic strain of Erwinia herbicola and IAA production in vitro. Can. J. Microbiol. 42: 586-592.

Brewer, C. A., Smith, W. K., and Vogelmann, T. C. 1991. Functional interaction between leaf trichomes, leaf wettability and the optical properties of water droplets. Plant Cell Environ. 14:955-962.

Burkhardt, J., and Hunsche, M. 2013. "Breath figures" on leaf surfacesformation and effects of microscopic leaf wetness. Front. Plant Sci. 4:422.

Burkhardt, J., Kaiser, H., Goldbach, H., and Kappen, L. 1999. Measurements of electrical leaf surface conductance reveal recondensation of transpired water vapour on leaf surfaces. Plant Cell Environ. 22:189-196.

Doan, H. K., and Leveau, J. H. J. 2015. Artificial surfaces in phyllosphere microbiology. Phytopathology 105:1036-1042.

Dulla, G., and Lindow, S. E. 2008. Quorum size of Pseudomonas syringae is small and dictated by water availability on the leaf surface. Proc. Natl. Acad. Sci. U.S.A. 105:3082-3087.

Fahrig, L. 2003. Effects of habitat fragmentation on biodiversity. Annu. Rev. Ecol. Evol. Syst. 34:487-515.

Farhan U1 Haque, M., Besaury, L., Nadalig, T., Bringel, F., Mutterer, J., Schaller, H., and Vuilleumier, S. 2017. Correlated production and consumption of chloromethane in the Arabidopsis thaliana phyllosphere. Sci. Rep. 7:17589.

Garin, G., Pradal, C., Fournier, C., Claessen, D., Houles, V., and Robert, C. 2018. Modelling interaction dynamics between two foliar pathogens in wheat: A multiscale approach. Ann. Bot. (Lond.) 121:927-940.

Giri, P., Taj, G., and Kumar, A. 2013. Comparison of artificial inoculation methods for studying pathogenesis of Alternaria brassicae (Berk.) Sacc. on Brassica juncea (L.) Czern. (Indian mustard). Afr. J. Biotechnol. 12: 2422-2426.

Gorb, S. N., Voigt, D., and Gorb, E. V. 2007. Visualisation of small fluid droplets on biological and artificial surfaces using the cryo-SEM approach. Mod. Res. Educ. Top. Microsc. 2:812-819.

Grinberg, M., Orevi, T., Steinberg, S., and Kashtan, N. 2019. Bacterial survival in microscopic surface wetness. eLife 8:e48508.

Hartley, S. E., Fitt, R. N., McLarnon, E. L., and Wade, R. N. 2015. Defending the leaf surface: Intra- and inter-specific differences in silicon deposition in grasses in response to damage and silicon supply. Front. Plant Sci. 6:35.

Innerebner, G., Knief, C., and Vorholt, J. A. 2011. Protection of Arabidopsis thaliana against leaf-pathogenic Pseudomonas syringae by Sphingomonas strains in a controlled model system. Appl. Environ. Microbiol. 77: 3202-3210.

Januszkiewicz, K., Mrozek-Niecko, A., and Rozanski, J. 2019. Effect of surfactants and leaf surface morphology on the evaporation time and coverage area of ZnIDHA droplets. Plant Soil 434:93-105.

Leben, C. 1988. Relative humidity and the survival of epiphytic bacteria with buds and leaves of cucumber plants. Phytopathology 78:179-185.

Leveau, J. H. J. 2009. Individual-based ecology of plant-associated bacteria. (Abstr.) Phytopathology 99:S155.

Leveau, J. H. J. 2019. A brief from the leaf: Latest research to inform our understanding of the phyllosphere microbiome. Curr. Opin. Microbiol. 49: 41-49.
Leveau, J. H. J., Hellweger, F. L., Kreft, J. U., Prats, C., and Zhang, W. W. 2018. The individual microbe: Single-cell analysis and agent-based modelling. Front. Microbiol. 9:2825

Leveau, J. H. J., and Lindow, S. E. 2001a. Appetite of an epiphyte: Quantitative monitoring of bacterial sugar consumption in the phyllosphere. Proc. Natl. Acad. Sci. U.S.A. 98:3446-3453.

Leveau, J. H. J., and Lindow, S. E. 2001b. Predictive and interpretive simulation of green fluorescent protein expression in reporter bacteria. J. Bacteriol. 183: 6752-6762.

Lindow, S. E., and Brandl, M. T. 2003. Microbiology of the phyllosphere. Appl. Environ. Microbiol. 69:1875-1883.

Maignien, L., Deforce, E. A., Chafee, M. E., Eren, A. M., and Simmons, S. L. 2014. Ecological succession and stochastic variation in the assembly of Arabidopsis thaliana phyllosphere communities. MBio 5:e00682-13.

Mechaber, W. L., Marshall, D. B., Mechaber, R. A., Jobe, R. T., and Chew, F. S. 1996. Mapping leaf surface landscapes. Proc. Natl. Acad. Sci. U.S.A. 93: 4600-4603.

Meier, U. 2001. Growth Stages of Mono- and Dicotyledonous Plants. BBCH Monograph, Blackwell, Berlin.

Monier, J. M., and Lindow, S. E. 2004. Frequency, size, and localization of bacterial aggregates on bean leaf surfaces. Appl. Environ. Microbiol. 70:346-355.

Monier, J.-M., and Lindow, S. E. 2005. Aggregates of resident bacteria facilitate survival of immigrant bacteria on leaf surfaces. Microb. Ecol. 49: 343-352.

Moyne, A.-L., Harris, L. J., and Marco, M. L. 2013. Assessments of total and viable Escherichia coli $\mathrm{O} 157: \mathrm{H} 7$ on field and laboratory grown lettuce. PLoS One 8:e70643

Moyne, A. L., Sudarshana, M. R., Blessington, T., Koike, S. T., Cahn, M. D., and Harris, L. J. 2011. Fate of Escherichia coli O157:H7 in field-inoculated lettuce. Food Microbiol. 28:1417-1425.

Olson, E. 2011. Particle shape factors and their use in image analysis-Part 1: Theory. J. GXP Compl. 15:85-96.

Parikh, A. N., Allara, D. L., Azouz, I. B., and Rondelez, F. 1994. An intrinsic relationship between molecular structure in self assembled $N$-alkylsiloxane monolayers and deposition temperature. J. Phys. Chem. 98:7577-7590.

Pathan, A. K., Bond, J., and Gaskin, R. E. 2010. Sample preparation for SEM of plant surfaces. Mater. Today 12:32-43.

Phillips, A. J. L. 1994. A comparison of methods for inoculating bean-plants with Elsinoe phaseoli and some factors affecting infection. Ann. Appl. Biol. 125:97-104.

Remus-Emsermann, M. N. P., De Oliveira, S., Schreiber, L., and Leveau, J. H. J. 2011. Quantification of lateral heterogeneity in carbohydrate permeability of isolated plant leaf cuticles. Front. Microbiol. 2:197.

Remus-Emsermann, M. N. P., and Leveau, J. H. J. 2010. Linking environmental heterogeneity and reproductive success at single-cell resolution. ISME J. 4: 215-222.

Remus-Emsermann, M. N. P., and Schlechter, R. O. 2018. Phyllosphere microbiology: At the interface between microbial individuals and the plant host. New Phytol. 218:1327-1333.

Remus-Emsermann, M. N. P., Tecon, R., Kowalchuk, G. A., and Leveau, J. H. J. 2012. Variation in local carrying capacity and the individual fate of bacterial colonizers in the phyllosphere. ISME J. 6:756-765.

Rowlandson, T., Gleason, M., Sentelhas, P., Gillespie, T., Thomas, C., and Hornbuckle, B. 2015. Reconsidering leaf wetness duration determination for plant disease management. Plant Dis. 99:310-319.

Ruinen, J. 1956. Occurrence of Beijerinckia species in the 'phyllosphere'. Nature 177:220-221.

Ryffel, F., Helfrich, E. J. N., Kiefer, P., Peyriga, L., Portais, J.-C., Piel, J., and Vorholt, J. A. 2016. Metabolic footprint of epiphytic bacteria on Arabidopsis thaliana leaves. ISME J. 10:632-643.

Schaeffer, P., Millet, J., and Aubert, J. P. 1965. Catabolic repression of bacterial sporulation. Proc. Natl. Acad. Sci. U.S.A. 54:704-711.

Schlechter, R. O., Miebach, M., and Remus-Emsermann, M. N. P. 2019 Driving factors of epiphytic bacterial communities: A review. J. Adv. Res. 19:57-65.

Schulte, A. J., Koch, K., Spaeth, M., and Barthlott, W. 2009. Biomimetic replicas: Transfer of complex architectures with different optical properties from plant surfaces onto technical materials. Acta Biomater. 5: 1848-1854

Scott, R. A., Thilmony, R., Harden, L. A., Zhou, Y. G., and Brandl, M. T. 2017. Escherichia coli $\mathrm{O} 157: \mathrm{H} 7$ converts plant-derived choline to glycine betaine for osmoprotection during pre- and post-harvest colonization of injured lettuce leaves. Front. Microbiol. 8:2436. 
Singh, S. 2016. Guttation: Mechanism, momentum and modulation. Bot. Rev. $82: 149-182$.

Soffe, R., Altenhuber, N., Bernach, M., Remus-Emsermann, M. N. P., and Nock, V. 2019a. Comparison of replica leaf surface materials for phyllosphere microbiology. PLoS One 14:e0218102.

Soffe, R., Bernach, M., Remus-Emsermann, M. N. P., and Nock, V. 2019b. Replicating Arabidopsis model leaf surfaces for phyllosphere microbiology. Sci. Rep. 9:14420.

Sun, M., Luo, C., Xu, L., Ji, H., Ouyang, Q., Yu, D., and Chen, Y. 2005. Artificial lotus leaf by nanocasting. Langmuir 21:8978-8981.

Sun, T. Z., Lazouskaya, V., and Jin, Y. 2019. Polydimethylsiloxane replicas efficacy for simulating fresh produce surfaces and application in mechanistic study of colloid retention. J. Food Sci. 84:524-531.

Taylor, P. 2011. The wetting of leaf surfaces. Curr. Opin. Coll. Int. Sci. 16: 326-334.

Tecon, R., and Leveau, J. H. J. 2012. The mechanics of bacterial cluster formation on plant leaf surfaces as revealed by bioreporter technology. Environ. Microbiol. 14:1325-1332.

Tian, J. F., Kannangara, D., Li, X., and Shen, W. 2010. Capillary driven low-cost V-groove microfluidic device with high sample transport efficiency. Lab Chip 10:2258-2264

Vacher, C., Hampe, A., Porté, A., Sauer, U., Compant, S., and Morris, C. 2016. The phyllosphere: Microbial jungle at the plant-climate interface. Annu. Rev. Ecol. Evol. Syst. 47:1-24.

van der Wal, A., and Leveau, J. H. J. 2011. Modelling sugar diffusion across plant leaf cuticles: The effect of free water on substrate availability to phyllosphere bacteria. Environ. Microbiol. 13:792-797.

Walters, D. R. 2006. Disguising the leaf surface: The use of leaf coatings for plant disease control. Eur. J. Plant Pathol. 114:255-260.
Wang, G., and Or, D. 2013. Hydration dynamics promote bacterial coexistence on rough surfaces. ISME J. 7:395-404.

Whipps, J. M., Lewis, K., and Cooker, R. C. 1988. Mycoparasitism and plant disease control. Pages 161-187 in: Fungi in Biological Control Systems. N. M. Burge, ed. Manchester University Press, Manchester, U.K.

Wilson, M., and Lindow, S. E. 1992. Relationship of total viable and culturable cells in epiphytic populations of Pseudomonas syringae. Appl. Environ. Microbiol. 58:3908-3913.

Wilson, M., and Lindow, S. E. 1994. Coexistence among epiphytic bacterialpopulations mediated through nutritional resource partitioning. Appl. Environ. Microbiol. 60:4468-4477.

Wu, W., Guijt, R. M., Silina, Y. E., Koch, M., and Manz, A. 2016. Plant leaves as templates for soft lithography. RSC Adv. 6:22469-22475.

Yang, D., Krasowska, M., Priest, C., Popescu, M. N., and Ralston, J. 2011. Dynamics of capillary-driven flow in open microchannels. J. Phys. Chem. C 115:18761-18769.

Zhang, B., Luo, Y., Pearlstein, A. J., Aplin, J., Liu, Y., Bauchan, G. R., Payne, G. F., Wang, Q., Nou, X., and Millner, P. D. 2014. Fabrication of biomimetically patterned surfaces and their application to probing plant-bacteria interactions. ACS Appl. Mater. Interfaces 6:12467-12478.

Zhang, M., Wu, J., Wang, L., Xiao, K., and Wen, W. 2010. A simple method for fabricating multi-layer PDMS structures for 3D microfluidic chips. Lab Chip 10:1199-1203.

Zheng, Y. M., Han, D., Zhai, J., and Jiang, L. 2008. In situ investigation on dynamic suspending of microdroplet on lotus leaf and gradient of wettable micro- and nanostructure from water condensation. Appl. Phys. Lett. 92:084106.

Zhu, H., Yu, Y., Ozkan, H. E., Derksen, R. C., and Krause, C. R. 2008. Evaporation and wetted area of single droplets on waxy and hairy leaf surfaces. Commun. Agric. Appl. Biol. Sci. 73:711-718. 